ARTIGO DE ATUALIZAÇÃO UPDATE ARTICLE

Palavras-chave:

integração vertical, práticas de gestão privada, análise envoltória de dados, eficiência

\section{Inovação na gestão hospitalar: análise dos resultados de eficiência de um hospital português integrado em uma unidade local de saúde}

\author{
Innovation in hospital management: analysis of efficiency \\ results of an portuguese hospital in a local health unit \\ Sérgio Farias ${ }^{1}$, Alexandre Morais Nunes² \\ DOI: 10.21115/JBES.v10.n3.p285-90
}

\section{RESUMO}

Objetivo: Verificar se a alteração do formato jurídico do hospital Amato Lusitano e a simultânea integração com a atenção primária gerou melhoria dos resultados de eficiência em seus serviços clínicos. Metodologia: Para avaliar os resultados de eficiência, utilizou-se à técnica da análise envoltória de dados, considerando como insumos os custos e como resultados a produção registrada de cada serviço clínico ao longo de um período de 15 anos. Os dados de custo e produção foram obtidos através do Gabinete de Apoio à Gestão e do Gabinete de Contabilidade do hospital. A amostra incluiu todos os serviços clínicos de internação, imagem e patologia clínica. Resultados: A mudança da gestão pública para a condição corporativa resultou em melhoria nos resultados de eficiência em 16 dos 17 serviços analisados apresentando um aumento médio de 19,0\%. Conclusões: A adoção da gestão empresarial e do modelo de unidade de saúde local tem sido benéfica para o desempenho dos serviços clínicos, com melhoria geral nos resultados de eficiência técnica.

\begin{abstract}
Objective: Verify that the change of the legal form of the Amato Lusitano Hospital and the simultaneous integration with primary care led to improved results in efficiency in their clinical services. Methodology: For evaluate the efficiency results, we used the technique of data envelopment analysis, considering as inputs the costs and as results the recorded production of each clinical service over a period of 15 years. Cost and production data were obtained through the Office of Management Support and the Accounting Office of the hospital. The sample included all clinical inpatient services, imagiology and clinical pathology. Results: The change of public management for corporate condition resulted in improvement in efficiency results, with 16 of the 17 services analyzed with an average increase of $19.0 \%$. Conclusions: The adoption of business management and the local health unit model has been beneficial to the performance of clinical services, overall improvement in technical efficiency results.
\end{abstract}

\footnotetext{
Recebido em: 08/10/2018. Aprovado para publicação em: 14/11/2018

1. Universidade da Beira Interior.

2. Universidade de Lisboa, Instituto Superior de Ciências Sociais e Políticas, Centro de Administração e Políticas Públicas (CAPP). Professor Auxiliar Convidado ISCSP-ULisboa. Coordenador Executivo da Pós-Graduação em Administração e Gestão da Saúde ISCSP-ULisboa.

Finaciamento: Não houve recebimentos de financiamento ou equipamento.

Correspondência: Sérgio Farias: e-mail: fariassergio1@hotmail.com - Telefone cellular: 00351963158647.
} 


\section{Introdução}

A sustentabilidade é um dos principais problemas dos sistemas de saúde predominantemente públicos, não só pelo seu impacto direto na população, mas também por seus efeitos indiretos na sociedade em geral, que afetam sobretudo a economia (Barros, 2013).

Em Portugal, os elevados gastos e a ineficiência do sector da saúde foram registrados na assistencia especializada em hospitais integrados no sector público administrativo, o que exigiu uma intervenção específica centrada na reforma do seu modelo de gestão. (Campos, 2008). Foi assim urgente promover a sustentabilidade na saúde, o que levou os sucessivos governos a repensar a estratégia de política de saúde. Daí, a adoção de uma reforma para promover a eficiência, sem comprometer a qualidade do atendimento, adotando práticas de gestão privada em hospitais públicos (Fernandes, 2014).

Em meados da década de 1990, com um momento de transformação na administração pública e com elavados gastos e ineficiências no sector da saúde, foram iniciadas diferentes experiências inovadoras de gestão nos hospitais públicos (Nunes \& Harfouche, 2015). Assim, sob a influência da perspectiva teórica da Nova Gestão Pública, foram adotadas práticas de gestão empresarial, processo conhecido como empresarização. Esse tipo de experimentalismo já tinha sido aplicado em outros países de influência anglo-saxônica e era conhecido sob o nome genérico de " corporisation of the public hospitals" (Nunes, 2017a).

Posteriormente, a publicação da Lei n.o 27/2002, de 8 de novembro, estabeleceu que os hospitais integrados no sistema de saúde poderiam ter um dos seguintes formatos jurídicos: hospitais integrados no Setor Publico Administrativo (SPA), hospitais empresas públicas (EPE) e hospitais Sociedade Anónima (SA). Três anos depois, o governo português decidiu melhorar os resultados fornecendo aos gerentes práticas de gestão. Essa decisão foi criticada dado que em tão pouco tempo se passou de práticas puramente públicas (até 2002) para um modelo de sociedade anônima (entre 2002 e 2004) e seguidamente para um formato de entidade pública empresarial muito próxima do setor privado (a partir de 2005). Em agosto de 2018, existiam 40 entidades da EPE: 11 hospitais (incluindo três institutos de oncologia), 21 centros hospitalares e 8 unidades de saúde locais (Portal SNS, 2018).

O modelo EPE estabeleceu a organização interna de hospitais, descentralização de poderes a nível local (organização), a abertura da regulação do setor e especialmente as carreiras profissionais e a harmonização das regras aplicou à administração pública os mecanismos característicos de direito comercial (Stoleroff \& Correia, 2008).

Ainda em 2005, com objetivo de preservar a viabilidade do sistema público de saúde, o governo fez um novo experi- mento baseado na fusão de hospitais com diferentes dimensões, capacidades e especificidades chamados de centros hospitalares (Fernandes \& Nunes, 2016; Nunes, 2017b). Esse processo de fusão e concentração de unidades hospitalares objetivou promover uma melhor gestão dos recursos técnicos e financeiros, sem comprometer a qualidade ou o acesso na assistência (Fernandes \& Nunes, 2016).

Ao mesmo tempo, e com o objetivo de promover a articulação entre a atenção primária à saúde e a atenção especializada (processo de integração vertical da atenção à saúde), sem prejuízo da introdução de práticas de gestão empresarial, foi implementado o modelo organizacional de Unidade Local de Saúde, que inclui a coordenação conjunta de ambos os níveis de atenção (primária e especializada).

O modelo ULS integra assim dois níveis de atenção à mesma estrutura de gestão, mas a negociação de contratos são distintas, com regras específicas para hospitais e outras para atenção primária (ACSS, 2009). Para além de ser um modelo inovador na administração de hospitais, a criação do ULS foi o precursor do sistema legal dos sistemas locais de saúde (SLS) criado um ano antes pelo Decreto-Lei n.o 156/99, de 10 de maio, para interligar os serviços e instituições de atenção primária à saúde, hospitais e outros serviços públicos de saúde, instituições privadas ou sociais, em proximidade geográfica próxima, para assegurar a continuidade dos cuidados de saúde e promover a racionalização da assistência em saúde.

O Hospital Amato Lusitano é um caso de inovação pois ao mesmo tempo que (2009), adotou diretamente o formato jurídico de empresa pública passou também ao modelo organizacional de unidade local de saúde, sem que os resultados de tal transformação fossem avaliados (Nunes \& Nunes, 2016). Foi por esse motivo, que é este estudo ganha relevância.

No presente artigo, realizou-se uma análise da eficiencia dos serviços clínicos com recurso a técnica da análise envoltória de dados. Para esta análise, foram usados dados financeiros e de produção registrados nos últimos 15 anos de atividade do Hospital Amato Lusitano.

\section{Método}

Um hospital é uma empresa multiproduto que produz, em diferentes quantidades, ensino, investigação, serviços comunitários, tratamento ambulatorial e tratamento hospitalar. A sua principal atividade é o tratamento de pacientes hospitalizados e é isto que os distingue de outras instituições de saúde (Carreira, 1999). A produção hospitalar por sua vez, evoluiu segundo as unidades de medida utilizadas que focalizaram duas principais linhas de produção hospitalar: serviços hospitalares e tratamentos ambulatoriais (Azevedo \& Mateus, 2014).

Com o objetivo de avaliar a evolução do formato juridico avaliou-se, no presente estudo, a evolução dos escores de 
eficiência técnica' dos serviços clínicos, de imagem e patologia clínica do Hospital Amato Lusitano, com recurso à Análise Envoltória de Dados.

A Análise Envoltória de Dados permite uma abordagem não paramétrica que utiliza programação matemática para definir a fronteira de eficiência. Assim, é possivel avaliar os escores de eficiência em função da razão entre a soma ponderada dos produtos (outputs) e a soma ponderada dos insumos (inputs) (Hollingsworth, Dawson, \& Maniadakis, 1999; Cooper, Seiford \& Tone, 2007).

Segundo a literatura, Análise Envoltória de Dados é o método mais utilizado para analisar a eficiência no setor da saúde (Cylus, Papanicolas \& Smith, 2016). O modelo permite definir a orientação do estudo que pode ser orientada para a minimização dos insumos (inputs) (representa o valor mínimo dos recursos para cada DMU) ou para os resultados (outputs) (maximização da produção, com base em um conjunto determinado de recursos iniciais) (Cooper et al., 2007).

Os modelos clássicos de Análise Envoltória de Dados desenvolvidos por Charnes, Cooper e Rhodes (CCR) em 1978 e Banker, Charnes e Cooper em 1984 (BCC) são essencialmente classificados de acordo com o retorno de escala, ou seja, Constante (Constant Return to Scale - CRS) ou Variavel (Variable Return to Scale - VRS) (Jubran). O modelo CRS avalia a eficiência total, identifica unidades eficientes e ineficientes e determina a que distância da fronteira de eficiência as unidades efetivas consideram um retorno constante à fronteira de escala. O modelo VRS foi então desenvolvido e inclui retornos variáveis de escala. (Azevedo \& Mateus, 2014). Basicamente, esse modelo identifica, dentre as unidades estudadas, aquela/aquelas que é mais eficiente, constituindo-a como uma fronteira, sendo todas as outras mais ineficientes, quanto maior a distância a essa fronteira (composta de DMU eficientes) (Cook \& Seiford, 2009; Cooper, Seiford, \& Tone, 2007).

Uma das limitações da Análise Envoltória de Dados foi encontrada nos casos em que várias DMUs estão no limite de eficiência, gerando o mesmo valor (escore $=1$ ). Para resolver essa questão, Andersen e Petersen (1993) propuseram um modelo capaz de medir a supereficiência, com o objetivo de classificar unidades eficientes para modelos radiais (por exemplo, CCR, BCC). (Anderson \& Petersen, 1993; Cooper et al., 2007). Assim, foi possível diferenciar as unidades eficientes e estabelecer um raking (é possível calcular entre as unidades com um rácio de eficiência $=1$, que são mais / menos efetivas) (Cooper et al., 2007; Zhu, 2001).

De acordo com a programação fracionária, o peso dado ás variáveis de inputs é $(v)\{i=1, \cdots, m\}$ e dos outputs é (u) $\{r=1, \cdots, s\}$

1 O conceito de eficiência técnica é definido como a situação em que é impossível que uma unidade de produção produza mais produtos com insumos que está a usar no know-how atual e na tecnologia existente (Cooper, Seiford, \& Tone, 2007). tal que

$\max \theta(v, u)=\frac{u_{1} y_{1 o}+u_{2} y_{2 o+\cdots+u_{s} y_{s o}}}{v_{1} x_{1 o}+v_{2} x_{2 o}+\cdots+v_{m} x_{m o}}$
$\frac{u_{1} y_{1 j}+\cdots+u y_{s j}}{v_{1} x_{1 o}+\cdots+v_{m} x_{m j}} \leq 1(j=1, \cdots, n)$
$v_{1}, v_{2}, \cdots, v_{m} \geq 0$
$u_{1}, u_{2}, \cdots, u_{s} \geq 0$

Com a fórmula anterior foi um problema com infinitas soluções, Charnes e Cooper (1978) propuseram uma resolução de programação linear (dual):

tal que

$\max \theta(\mu, v)=\mu_{1} y_{1 o}+\cdots+\mu_{s} y_{s o}$
$v_{1} x_{1 o}+\cdots+v_{m} x_{m o}=1$
$\mu_{1} y_{1 j}+\cdots+\mu_{s} y_{s j} \leq v_{1} x_{1 j}+\cdots+v_{m} x_{m j} \quad(j=1, \cdots, n)$
$v_{1}, v_{2}, \cdots, v_{m} \geq 0$
$\mu_{1}, \mu_{2}, \cdots, \mu_{s} \geq 0$

Tendo em conta estes objetivos, decidiu-se aplicar um modelo de CCR orientado para o output.

$\max (n, \mu) \quad n$
$x_{o}-X \mu \geq 0$
$n y_{o}-Y \mu \leq 0$
$\mu \geq 0$

Devido à homogeneidade dos resultados, optamos pelo conceito de supereficiência de Andersen e Petersen (2003).

$\theta^{*}=\min \left(\theta, \lambda, s^{-}, s^{+}\right) \theta-\varepsilon e s^{+}$
$\theta x_{o}=\sum_{j=1, \neq 0}^{n} \lambda_{j} x_{j}+s^{-}$
$y_{o}=\sum_{j=1, \neq 0}^{n} \lambda_{j} y_{j}+s^{+}$

A avaliação da eficiência hospitalar, entre 2002 e 2017, utilizou-se, para este estudo, como:

- Inputs, os custos totais (diretos e indiretos) efetuados em cada departamento do Hospital Amato Lusitano;

- Outputs, os doentes saídos do internamento, número total de consultas, número total 
de cirurgias e número de sessões do dia no hospital. No caso dos MCDT's, os resultados considerados foram radiografias convencionais, tomografia axial computadorizada, mamografia e ultrassonografia; e análises de bioquímica, hematologia, microbiologia e imunologia/virologia.

A amostra do estudo foi os serviços clínicos do hospital (tabela 1).

Os dados foram fornecidos pelo Hospital Amato Lusitano, através do seu Serviço de Gestão Financeira e Gabinete de Apoio à Gestão.

Tabela 1. Serviços clínicos incluidos no estudo

\begin{tabular}{|c|c|}
\hline \multirow{10}{*}{ Área médica } & Especialidade \\
\hline & Cardiologia \\
\hline & Gastroenterologia \\
\hline & Medicina Interna \\
\hline & Nefrologia \\
\hline & Neurologia \\
\hline & Pediatria/Neonatologia \\
\hline & Pneumologia \\
\hline & Psiquiatria (agudos) \\
\hline & Dermatovenerologia \\
\hline \multirow{6}{*}{ Área cirurgica } & Cirurgia Geral \\
\hline & Oftalmologia \\
\hline & Ginecologia/Obstetrícia \\
\hline & Otorrinolaringologia \\
\hline & Ortopedia \\
\hline & Urologia \\
\hline \multirow{3}{*}{$\begin{array}{l}\text { Áreas } \\
\text { complementares }\end{array}$} & $\begin{array}{l}\text { Meios Complementares de } \\
\text { Diagnostico e Terapêutica }\end{array}$ \\
\hline & Imagiologia \\
\hline & Patologia Clinica \\
\hline
\end{tabular}

Fonte: Elaboração própria

\section{Resultados}

Para verificar se a transição para o formato EPE, em 2009, gerou maior eficiencia nos todos os serviços clínicos do Hospital Amato Lusitano em comparação com o período anterior, foi realizada uma avaliação da eficiência técnica dos serviços utilizando a Análise Envoltória de Dados, consideranda uma avaliação global da variação média na eficiência técnica registrada durante o período do estudo. A avaliação global em termos da variação média de eficiência registrada a cada ano nos serviços durante o período em estudo é mostrada na Figura 1.

Para medir o efeito da transição do formato jurídico ocorrida em 2009, foram considerados os resultados médios de eficiência em dois momentos diferentes: o primeiro, no período anterior à mudança de status (2002-2008) e o segundo, nos anos seguintes (2010-2017)

Para o período de 2002-2008, verificou-se uma eficiência técnica média de 0,66 , enquanto que para o período de 2010-2017, a eficiência técnica média foi de 0,85.

Comparando os dados nos períodos considerados, observa-se que com a transição para o status da sociedade, todos os serviços, exceto Neurologia (correspondendo a 94,1\%), melhoram sua eficiência técnica (Figura 2).

\section{Discussão}

No que respeita à transição para o estatuto E.P.E. a partir de 2009, todos os serviços clínicos do Hospital Amato Lusitano foram mais eficientes do que no período anterior, com um aumento médio da eficiência técnica de 19,0\%, no período correspondente ao estatuto da empresarial, em relação ao anterior, o formato do Sector Público Administrativo.

Os resultados obtidos neste estudo são consistentes com os resultados dos estudos que concluíram que E.P.E. têm valores de eficácia técnica mais elevados do que os hospitais SPA; Harfouche (2012), em seu estudo, constatou que os

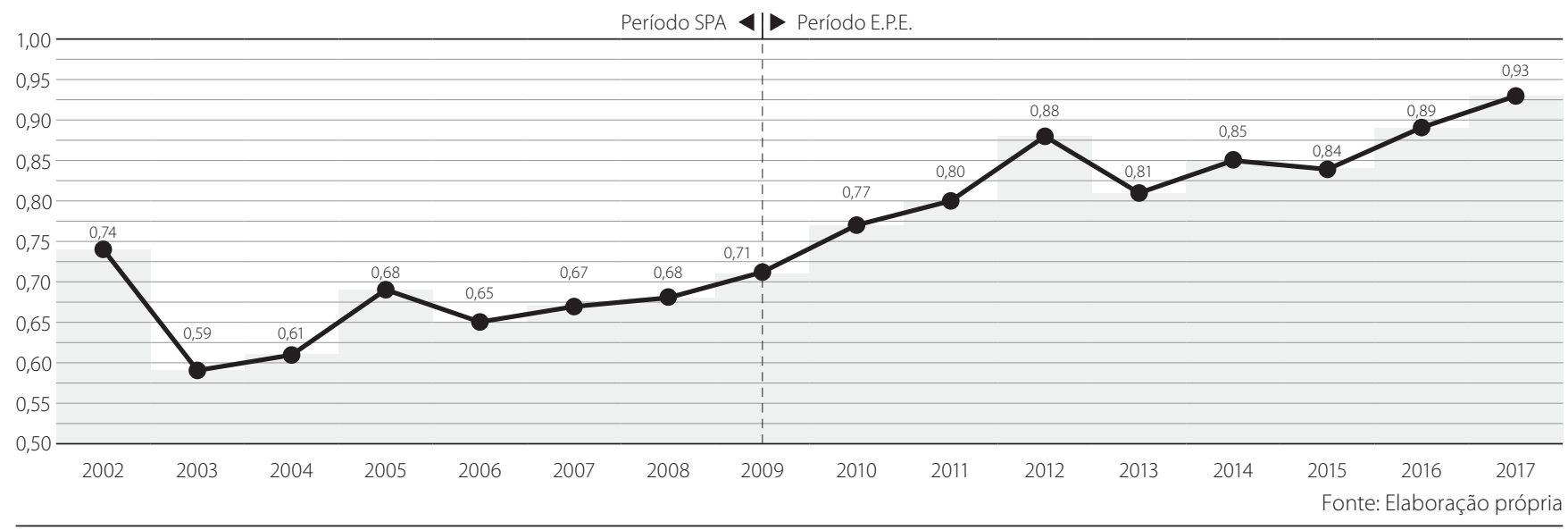

Figura 1. Evolução dos escores de eficiência média registados no Hospital Amato Lusitano, entre 2002 e 2017 


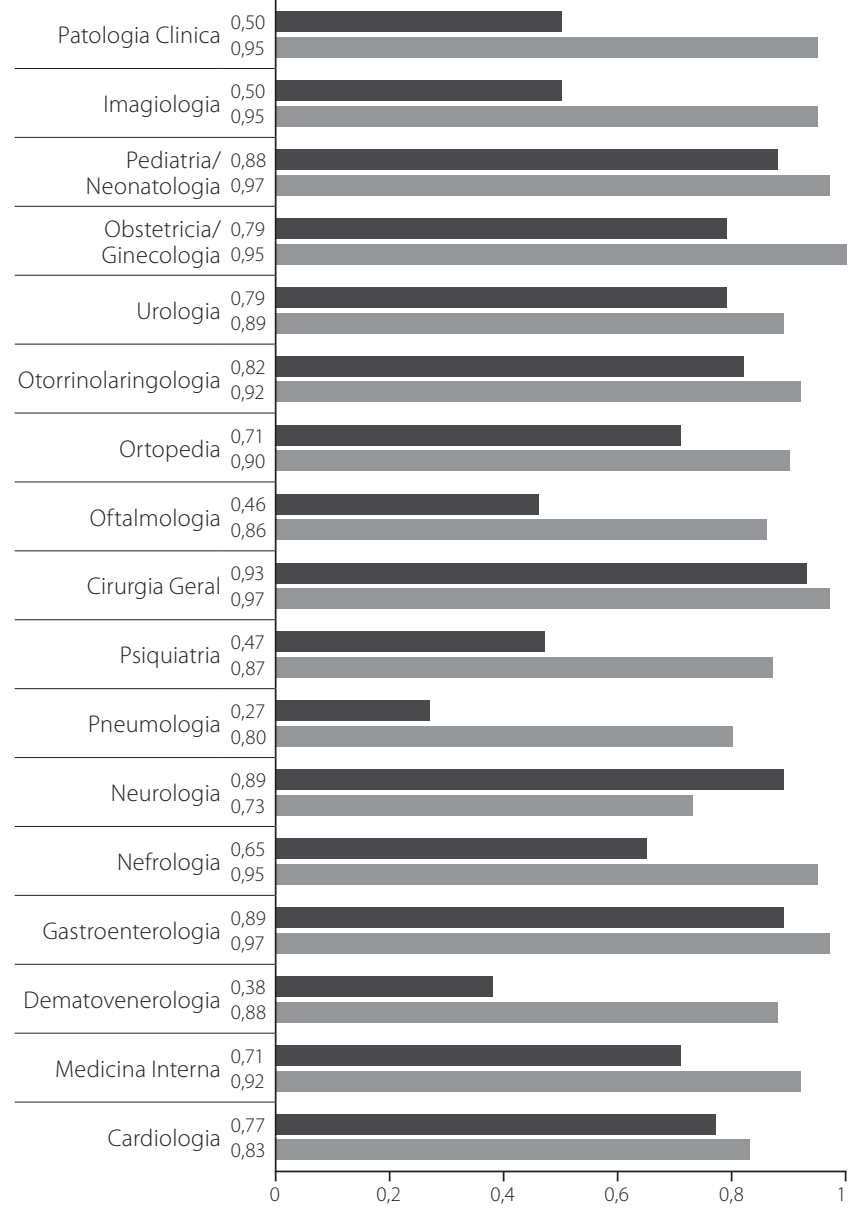

2002-2008 2010-2017

Fonte: Elaboração própria

Figura 2. Resultados de cada serviço nos períodos 2002-2008 e 2010-2017.

hospitais com práticas empresariais atingiram ganhos de eficiências, como neste estudo, e Nunes (2016), que também concluiu, numa perspectiva de longo prazo, que a gestão empresarial de hospitais públicos forneceu-lhes resultados mais eficazes. Ferrier e Valdmanis (2004) nos Estados Unidos da América descobriram que as fusões hospitalares trazem maior eficiência. Chang e Cheng (2004), em Taiwan, descobriram que os hospitais públicos são menos eficientes do que os hospitais privados.

\section{Conclusões}

Ao analisar os escores de eficiência, verificou-se que durante o período 2002-2008, correspondente ao estatuto integrado no setor público, a eficiência técnica média do Hospital Amato Lusitano foi de 0,66. No entanto, para o período 2010-2017, correspondente à transição para o modelo de unidade local de saúde com formato jurídico empresarial, a eficiência técnica média foi de 0,85. Assim, se verificou que o Hospital Amato Lusitano registou um ganho de eficiência de cerca de 19\%.
De acordo com os resultados obtidos, foi possível concluir que a transição do status legal do SPA para o E.P.E. em particular, melhorou os resultados de eficiência em 94,1\% dos serviços incluídos neste estudo.

\section{Referencias Bibliográficas}

ACSS - Administração Central do Sisitema de Saúde (2009). Unidades Locais de Saúde, Modalidade de Pagamento - Unidade Operacional de Financiamento e Contratualização. Lisboa: ACSS. Disponivel http:// www2.acss.min-saude.pt/Portals/0/modalidadeULS09.pdf.

Andersen $\mathrm{P}$, petersen N. A procedure for ranking efficient units in data envelopment analyses. Management Science. 1993; 39(10), 1261-1264.

Azevedo H, Mateus C. Economias de escala e de diversificação: uma análise da bibliografia no contexto das fusões hospitalares. Revista Portuguesa de Saúde Pública. 2014; 32(1), 106-117.

Barros PP. Economia da Saúde. Coimbra: Almedina; 2013.

Campos A. Reformas da saúde - o fio condutor. Coimbra: Edições Almedina; 2008.

Carreira CM. Economias de Escala e de Gama nos Hospitais Públicos Portugueses: uma aplicação da função de custo variável translog. Estudos de Economia. 1999; 19(3), 273-294.

Chang H, Cheng M. Hospital ownership and operating efficiency: Evidence from Taiwan. European Journal of Operational Research. 2004; 159(2), $513-27$.

Charnes A, Cooper W, Rhodes E. Measuring the efficiency of decision making units. European Journal of Operational Research. 1978; 2, 429-444.

Cook W, Seiford L. Data envelopment analysis (DEA)-thirty years on. European. Journal of Operational Research. 2009; 192(1), 1-17.

Cooper W, Seiford L, Tone, K. Data envelopment analysis: a comprehensive text with models, applications, references and DEA-solver software(2nd Edition). Reino Unido: Springer; 2007.

Cylus J, Papanicolas I, Smith PC. Health system efficiency - How to make measurement matter for policy and management. Copenhaga: Organização Mundial da Saúde; 2016.

Farrel. The measurement of productive efficiency. Journal of the Royal Statistical Society. 1957; 120(3), 253-281.

Fernandes AC, Nunes AM. Os Hospitais e a Combinação Público-Privado no Sistema de Saúde Português. Acta Medica Portuguesa; 2016. 29(3), 217-223.

Fernandes AC. A combinação público-privado. In Campos, A. \& Simões, J. (Coord). 40 anos de abril na saúde(pp. 213-231). Coimbra: Almedina; 2014.

Ferrier $\mathrm{G}$, Valdmanis V. Do mergers improve hospitalproductivity? Journal of the Operational Research Society. 2004; 55, 1071-1080.

Hollingsworth B, Dawson P, Maniadakis N. Efficiency measurement of health care: a review of non-parametric methods and applications. Health care management science. 1999; 2, 161-172.

Nunes AM. Análise da produtividade da política de fusão de unidades hospitalares em Portugal integradas no Serviço Nacional de Saúde. Jornal Brasileiro de Economia da Saúde. 2017b; 9(1), 93-9.

Nunes AM. Reformas na Gestão Hospitalar: Análise dos efeitos da empresarialização (tese de Doutoramento em Ciências Sociais na Especialidade de Administração da Saúde). Lisboa: Instituto Superior de Ciências Sociais e Políticas da Universidade de Lisboa; 2016.

Nunes AM, Harfouche APA Reforma da Administração Pública Aplicada ao Setor da Saúde: A Experiência Portuguesa. Revista de Gestão em Sistemas de Saúde, julho/dezembro. 2015; (4), 1-8. 
Nunes AM, Nunes ML. A saúde em Portugal: um olhar sobre o Distrito de Castelo Branco. Castelo Branco: RVJ - Editores; 2016.

Nunes AM. Do mundo empresarial à gestão hospitalar: a experiência portuguesa. Revista FAE. 2017a; 20(1), 93 - 104.

Portal SNS, 2018. Entidades de Saúde. Disponivel emhttps://www.sns.gov.pt/ institucional/entidades-de-saude/

Portugal. Decreto-Lei n.o 156/99, de 10 de maio.

Portugal. Decreto-Lei n. ${ }^{3}$ 318/2009.

Portugal. Decreto-Lei n. 93/2005.

Portugal. Diário da República n. ${ }^{109 / 2005 .}$
Portugal. Lei n. ${ }^{\circ}$ 27/2002.

Portugal. Memorando de entendimento sobre as condicionalidades de política económica. Programa de assistência financeira UE/FMI/BCE. Request for a Three-Year Arrangement Under the Extended Fund Facility, IMF Country Report No. 11/127, June 2011. Disponível em https:// www.portugal.gov.pt/media/371372/mou_pt_20110517.pdf

Stoleroff A, Correia T. Sindicalismos no contexto de reforma dos serviços públicos em Portugal: o setor hospitalar. VI Congresso Português de Sociologia, Mundos Sociais: Saberes e Práticas; 2008.

Zhu J. Super-Efficiency and DEA Sensitivity Analysis. European Journal of Operational Research. 2001; 129(2), 443-455. 